\title{
Desempenho agronômico e diversidade genética de linhagens de feijão- caupi nas condições do Recôncavo da Bahia
}

\author{
Zalmar Santana Gonçalves ${ }^{a^{*}}$, Lucas Kennedy Silva Lima ${ }^{a} \bullet$
}

a Embrapa Mandioca e Fruticultura, Brasil

*Autor correspondente (zalmarufrb@hotmail.com)

\section{N F O}

\section{Keyworks}

agronomic characterization

Vigna unguiculata $\mathrm{L}$.

walp

production

genetic variability

\begin{abstract}
A B S T R A C T
Agronomic performance and genetic diversity of cowpea lines under conditions of Recôncavo da Bahia. Cowpea is among the main subsistence crops for small farmers in the North and Northeast of Brazil. However, its productivity is low compared to other regions of the country, and may be associated with the use of non-adapted cultivars. Thus, the objective was to identify the most productive cowpea lines and adapted to the conditions of the Recôncavo da Bahia. The experiment was carried out in Cruz das AlmasBA, located in Recôncavo da Bahia, consisting of 16 lines and four cultivars of cowpea totaling 20 treatments that were distributed in a randomized block design and four replications. At the moment when all the varieties in the plot were dry, the harvest was carried out and then the evaluation of the agronomic characteristics was carried out. average length of the pods; weight of five pods; grain weight in five varieties, number of grains in five pods and average number of grains per pod. The data were subjected to analysis of variance and their means were grouped by the Scott-Knott test, in order to complement the information and demonstrate the genetic variability between strains. Multivariate analysis was performed. There were differences between the strains for the five characteristics evaluated and from the analysis of main components and cluster analysis and heatmap, the formation of four groups was observed, with emphasis on the strains MNC03-737F-5-9 and MNC03-736F-7, which exhibited agronomic potential in relation to the other lines and cultivars evaluated, and are therefore recommended for the production of cowpea under the conditions of the Recôncavo the Bahia.
\end{abstract}

\section{R E S U M O}

O feijão-caupi [Vigna unguiculata (L.) Walp] está entre as principais culturas de subsistência para pequenos agricultores do Norte e Nordeste do Brasil. Contudo, sua produtividade é baixa em comparação a outras regiões do país, podendo estar associada à utilização de cultivares não adaptadas. Assim, objetivouse identificar as linhagens de feijão-caupi mais produtivas e adaptadas às condições do Recôncavo da Bahia. O experimento foi conduzido em Cruz das Almas-BA, localizada no Recôncavo da Bahia, sendo constituídas por 16 linhagens e quatro cultivares de feijão-caupi, totalizando 20 tratamentos que foram distribuídas em delineamento experimental em blocos casualizados e quatro repetições. No momento em que todas as vargens da parcela se encontravam secas foi realizada a colheita e em seguida foi realizada a avaliação das características agronômicas, comprimento médio das vagens; massa de cinco vagens; massa dos grãos em cinco vargens, número de grãos em cinco vagens e número médio de grãos por vagem. Os dados foram submetidos à análise de variância e suas médias foram agrupadas pelo teste Scott-Knott, visando complementar as informações e demonstrar a variabilidade genética entre as linhagens foi realizada análise multivariada. Houve diferenças entre as linhagens para as cinco características avaliadas e a partir da análise de componentes principais e análise de agrupamento e heatmap foi observada a formação de quatro grupos com destaque para as linhagens MNC03-737F-5-9 e MNC03-736F-7, que exibiram potencial agronômico em relação às demais linhas e cultivares avaliadas, sendo, portanto, recomendadas para produção de feijão-caupi nas condições do Recôncavo da Bahia. 


\section{INTRODUÇÃO}

Com o crescente aumento da população mundial, a disponibilidade de alimentos para atender às necessidades nutricionais tem se tornado cada vez mais escassa, sobretudo nos países subdesenvolvidos com baixa renda per capta. A tendência de agravamento desse problema é eminente diante das mudanças climáticas que estão alterando os processos produtivos em todo o mundo, demandando novas tecnologias para contornar os efeitos deletérios do aquecimento global (Agovino et al., 2019; Bocchiola et al., 2019). Assim, estudos direcionados para a seleção de variedades adaptadas às condições ambientais desfavoráveis e que promova manutenção da produtividade das culturas são imprescindíveis. Além disso, atenção maior deve ser dada aos cereais proteicos, que correspondem entre 10 a $20 \%$ da proteína consumida pelos países mais pobres do mundo (Akibode e Maredia, 2012).

O feijão-caupi [Vigna unguiculata (L.) Walp.] pertencente à família Fabaceae, utilizado na alimentação humana e animal em diversos países da América Latina, e Continentes Africano e Asiático, servindo principalmente como fonte de proteínas (23-25\%) e carboidratos entre 50-67\% (Devi et al., 2015). No Brasil, a cultura é explorada em todas as regiões, mas os principais estados produtores na safra 2019/2020 foram Mato Grosso, Ceará, Bahia e Piauí, com produção de 150,6, 144,9, 109,6 e 85,7 mil t ha ${ }^{-1}$, respectivamente, correspondendo a quase $70 \%$ da produção nacional. No Nordeste o feijão-caupi é explorado principalmente pelos pequenos agricultores do Semiárido devido à sua adaptação às condições edafoclimáticas da região em relação às outras espécies da família Fabaceae e por ser utilizada em diversos pratos típicos (Araújo et al., 2019; Dantas et al., 2019; Silva et al., 2019). Contudo, o feijãocaupi apresenta baixa produtividade nesta região $\left(470 \mathrm{~kg} \mathrm{ha}^{-1}\right)$ principalmente quando comparado a produtividade média da parte Centro-Oeste do Brasil que é de $1.200 \mathrm{t} \mathrm{ha}^{-1} /$ ano (Barros et al., 2013; CONAB, 2021), o que em parte, está associada à utilização de cultivares não recomendadas para essa região (Barros et al., 2013; Araújo et al., 2019). Desse modo, a utilização de material genético não adaptado pode levar ao baixo rendimento da cultura, impactando diretamente na rentabilidade financeira da atividade agrícola, comprometendo o investimento em insumos e a estabilidade de toda a cadeia produtiva.

Diversas pesquisas foram conduzidas com a finalidade de selecionar linhagens e/ou cultivares de feijão-caupi adaptadas as condições do Semiárido brasileiro (Freire Filho et al., 2007; Santos e Lima, 2015; Araújo et al., 2019), sendo reportada ampla variação no vigor e produção dos genótipos em função do local e do manejo utilizado na condução da cultura. Contudo, os estudos voltados para as condições do Recôncavo da Bahia, Brasil são escassos, sendo necessária a seleção de genótipos adaptados a esta região, com a finalidade de subsidiar os programas de melhoramento genético da variedade caupi ou para recomendação de cultivares mais adaptadas e altamente produtivos.

Neste sentido, objetivou-se identificar as linhagens de feijão-caupi mais produtivas, adaptadas e com possível aceitação comercial pelos agricultores e consumidores da região do Recôncavo baiano e que futuramente poderão ser incorporadas em ensaios de competição de variedades em outras regiões do Brasil.

\section{MATERIAL E MÉTODOS}

\section{Local do experimento e preparo da área}

O experimento foi conduzido no campo expimental da Universidade Federal do Recôncavo da Bahia-UFRB, Campus Cruz das Almas-BA, situado a $12^{\circ} 40^{\prime} 19^{\prime \prime}$ de Latitude Sul, 39 $06^{\prime} 22^{\prime \prime}$ de Longitude Oeste, e $220 \mathrm{~m}$ de altitude. O clima é tropical quente e úmido, Aw a Am, segundo a classificação de Koppen, com temperaturas médias anuais de $24,5{ }^{\circ} \mathrm{C}$ e umidade relativa em torno de $70 \%$. O solo da área experimental é classificado como Latossolo Amarelo Distrófico profundo, considerado como do tipo Franco argilo-arenoso (com $84 \%$ de grau de dispersão de argila e caracterizados por solos de baixa fertilidade) de acordo com a caracterização físico-hídrica de solos da área do Centro Nacional de Pesquisa de Mandioca e Fruticultura (Souza e Souza, 2001). Para as condições do Recôncavo da Bahia em que a concentração das chuvas acontece entre os meses de maio a julho, o plantio ocorreu no mês de junho e a colheita foi realizada 90 dias após a semeadura no período seco.

Quatro semanas antes do plantio foi realizada uma aração, seguida de duas gradagens e correção do solo com uso de calcário Dolomítico, de acordo com a análise de solo e a partir da Recomendação de Calagem e Adubação para o Cultivo do feijão (comunicado técnico, 2009). A adubação fosfatada $\left(70 \mathrm{~kg} \mathrm{ha}^{-1}\right)$ foi realizada no momento do plantio e duas adubações nitrogenadas $\left(80 \mathrm{~kg} \mathrm{ha}^{-1}\right)$ foram realizadas diretamente ao solo com 15 e 30 dias após a emergência das linhagens, assim como o fornecimento de cloreto de potássio $\left(60 \mathrm{~kg} \mathrm{ha}^{-1}\right)$, sem irrigação 'tipo sequeiro' seguindo as recomendações técnicas para a cultura (Pereira et al., 2015). 


\section{Material vegetal e condução experimental}

Os tratamentos foram constituídos por 16 linhagens de feijão-caupi e 4 cultivares comerciais como controle, sem qualquer tipo de tratamento prévio oriundas do Banco Ativo de GermoplasmaBAG da Embrapa Meio-Norte, situada em Teresina-PI, Brasil (Tabela 1). O experimento foi conduzido em delineamento em blocos casualizados, com 20 tratamentos e quatro repetições de 40 plantas na parcela útil. As parcelas de $2 \mathrm{~m}$ x $5 \mathrm{~m}$ continham quatro fileiras de $5 \mathrm{~m}$ de comprimento, tendo como áreas úteis as duas fileiras centrais. $\mathrm{O}$ espaçamento entre fileiras foi de $0,50 \mathrm{~m}$ e $0,25 \mathrm{~m}$ entre plantas, resultando em 20 plantas por linha.

A colheita foi realizada uma única vez, acontecendo quando todas as vagens da parcela estavam secas, não necessitando de uma segunda colheita. A secagem das vagens com os grãos foi realizada ao sol por 2 a 3 dias, reduzindo a umidade para $10-12 \%$, todos os grãos foram separados das vagens através de abertura manual. A palhada foi separada dos grãos com utilização de uma peneira, e logo após isso, foram contadas, pesadas e realizadas todas as outras variaveis do trabalho.

Tabela 1 - Linhagens de feijão-caupi e seus respectivos parentais, avaliados quanto as suas principais características agronômicas, em Cruz das Almas-Bahia, Brasil.

\begin{tabular}{ccc}
\hline Código das Linhagens & Parentais / Procedência das linhagens & $\begin{array}{c}\text { Subclasse } \\
\text { comercial }\end{array}$ \\
\hline MNC02-675F-4-9 & TE97-309G-24 xTE96-406-2E-28-2 & Mulato \\
MNC02-675F-4-2 & TE97-309G-24 x TE96-406-2E-28-2 & Mulato \\
MNC02-675F-9-2 & TE97-309G-24 x TE96-406-2E-28-2 & Mulato \\
MNC02-675F-9-3 & TE97-309G-24 x TE96-406-2E-28-2 & Mulato \\
MNC02-676F-3 & TE97-309G-24 x EV x 91-2E-2 & Mulato \\
MNC02-682F-2-6 & TE96-282-226 x MNC00-519-7-1-1 & Branco \\
MNC02-683F-1 & BR9-LONGA x TE96-282-22G & Branco \\
MNC02-684F-5-6 & TE96-282-22G x TE96-406-2E-28-2 & Branco \\
MNC03-725F-3 & MNC01-627D-65-1 x TE99-499-1F-2-1 & Branco \\
MNC03-736F-7 & TE97-309G-24 X IT90N-284-2) x TE96-282-22G & Branco \\
MNC03-737F-5-1 & TE96-282-22G x IT81D-1332 & Branco \\
MNC03-737F-5-4 & TE96-282-22G x IT81D-1332 & Branco \\
MNC03-737F-5-9 & TE96-282-22G x IT81D-1332 & Branco \\
MNC03-737F-5-10 & TE96-282-22G x IT81D-1332 & Branco \\
MNC03-737F-5-11 & TE96-282-22G x IT81D-1332 & Branco \\
MNC03-737F-11 & TE96-282-22G x IT81D-1332 & Branco \\
\hline Código das Cultivares & Parentais / Procedência das Cultivares & Subclasse \\
\hline BRS-TUMUCUMAQUE & TE96-282-22G x IT87D-611-3 & Branco \\
BRS-CAUAMÉ & TE93-210-13F x TE96-282-22G & Branco \\
BRS-ITAIM & MNC01-625E-10-1-2-5 x MNC99-544D-10-1-2-2 & Branco \\
BRS-GUARIBA & IT85-2687 x TE87-98-8G & Branco \\
\hline
\end{tabular}

\section{Variáveis e análise dos dados}

Foram avaliadas cinco características agronômicas, a citar: comprimento médio das vagens - COMPV $(\mathrm{cm})$; massa de cinco vagens M5V (g); massa dos grãos em cinco vagens MG5V (g), onde os grãos foram uniformizados quanto a sua umidade que variou entre $10-12 \%$, e pesados em balança Analítica Digital de Precisão; número de grãos em cinco vagens - NG5V e número médio de grãos por vagem- NGV, contagem obtida da média de cinco vagens tiradas ao acaso. Os dados foram submetidos à análise de variância (ANAVA) quando significativo, as médias das 20 linhagens foram agrupadas pelo teste Scott-Knott com 5\% de probabilidade.

Para complementar as análises de variância e teste de média, os dados agronômicos foram submetidos à análise por agrupamento, por meio da medida de dissimilaridade utilizando a distância de 
Gower como método de agrupamento Unweighted Pair-Group Method Using an Arithmetic Average (UPGMA) juntamente com a análise de agrupamento foi gerado heatmap para as 20 linhagens em função dos cinco caracteres biométricos, visando indicar o melhor grupo e a relação das linhagens e cultivares com as variáveis.

Por fim, os dados foram submetidos à análise de componentes principais (PC) e os genótipos foram plotados em relação aos dois primeiros componentes ( $\mathrm{PC} 1$ e $\mathrm{PC} 2$ ). $\mathrm{O}$ número de grupo foi definido com base na análise de Cluster visualizada no heatmap e dispersão dos genótipos nos dois primeiros componentes (PC1 e PC2). A correlação de Pearson também foi utilizada visando determinar a relação entre os caracteres biométricos e a significância entre os pares de correlação determinado pelo valor de $(P)$, na mesma figura também foram apesentados histogramas com a curva de distribuição normal para cada característica e gráficos de dispersão de pontos entre as variáveis. Todas as análises foram realizadas utilizando programa estatístico $\mathrm{R}$ versão (v.4.0.0) com os pacotes 'Agricolae', 'ExpDes.pt', 'SuperHetmap', 'ggplot' e 'Performance Analytics', (R Development Core Team, 2020)

\section{RESULTADOS}

Houve variação entre as linhagens de feijãocaupi cultivadas nas condições do Recôncavo da Bahia para as cinco variáveis analisadas, com variação de $1 \%(\mathrm{p} \leq 0,01)$ para o comprimento médio das vagens (COMPV), massa de cinco vagens (M5V) e massa dos grãos de cinco vagens (MG5V) (Tabela 2).

Tabela 2 - Resumo da análise de variância para comprimento médio das vagens (COMPV) massa de cinco vagens (M5V), massa dos grãos em cinco vagens (MG5V), número de grãos em cinco vagens (NG5V) e número médio de grãos das vagens $(\mathrm{NGV})$ para 16 linhagens de feijão-caupi e quatro cultivares comerciais avaliadas nas condições do Recôncavo da Bahia, Brasil.

\begin{tabular}{ccccccc}
\hline \multirow{2}{*}{ FV } & GL & \multicolumn{7}{c}{$\mathbf{Q M}$} \\
\cline { 3 - 7 } & & $\begin{array}{c}\text { COMPV } \\
(\mathbf{c m})\end{array}$ & $\begin{array}{c}\text { M5V } \\
(\mathbf{g})\end{array}$ & $\begin{array}{c}\text { MG5V } \\
(\mathbf{g})\end{array}$ & NG5V & NGV \\
\hline Bloco & 3 & $4,45^{* *}$ & $5,52^{\text {ns }}$ & $2,13^{\text {ns }}$ & $95,52^{*}$ & $9,59^{*}$ \\
Linhagens & 19 & $5,14^{* *}$ & $15,93^{* *}$ & $8,43^{* *}$ & $51,5^{*}$ & $12,03^{*}$ \\
Erro & 59 & 1,55 & 4,76 & 3,28 & 77,00 & 7,89 \\
C.V. (\%) & & 7,33 & 16,64 & 7,38 & 17,35 & 9,91 \\
Média geral & & 16,93 & 13,11 & 10,41 & 50,58 & 10,01 \\
\hline
\end{tabular}

${ }_{\text {ns }}$ não significativo, ${ }^{* *} \mathrm{e}^{*}$ significativo a 1 e $5 \%$, respectivamente pelo teste de F, (C.V. \%) Coeficiente de Variação.

A variação entre as linhagens de feijão-caupi cultivadas nas condições do Recôncavo da Bahia evidenciam as diferenças genotípicas, portanto, a possibilidade de ganhos genéticos de seleção, como reportado em outros trabalhos de mesma natureza (Locatelli et al., 2014; Santos et al., 2014; Sousa et al., 2015; Araújo et al., 2019) e a recomendação de cultivares adaptadas as condições edafoclimáticas deste estudo.

Para o coeficiente de variação, seus valores variaram entre $7,33 \%$ a $17,35 \%$ para comprimento médio das vagens e número de grãos em cinco vagens respectivamente, valores análogos aos trabalhos de (Teixeira et al., 2010; Silva e Neves 2011; Barros et al., 2013; Santos et al., 2014 e Araújo et al., 2019).

O comprimento médio das vagens (COMPV) variou de 15,27-20,00 $\mathrm{cm}$ entre as linhagens MNC03-737F-5-9 e MNC03-736F-7, respectivamente, sendo observada a formação de dois grupos para essa característica (Tabela 3 ). Este comportamento foi semelhante ao reportado por Torres et al. (2015) avaliando genótipos de feijãocaupi na região Centro-Oeste do Brasil, indicando que apesar da variação nas condições edafoclimáticas entre os ambientes é possível selecionar linhagens adaptadas a diferentes condições, enaltecendo a importância da seleção local de genótipos adaptados para subsidiar os programas de melhoramento ou para recomendação de cultivares produtivas e adaptadas às principais pragas e doenças comuns na região do estudo.

Por outro lado, os valores de COMPV apresentados por Silva e Neves (2011), Santos e Lima (2015) e Araújo et al. (2019), foram maiores em relação aos alcançados neste estudo. Podendo estar relacionado a temperaturas mais baixas, umidade relativa alta e índice pluviométrico mais elevado na região do Recôncavo, o que desfavoreceu o desempenho dessas linhagens que são oriundas do Banco Ativo de GermoplasmaBAG da Embrapa Meio-Norte, situada em Teresina-PI e provavelmente adaptadas a essas condições, contudo, algumas linhagens como MNC03-736F-7 e MNC02-675F-4-2 se destacaram em relação ao conjunto avaliado e exibiram potencial produtivo. Conforme Bastos, Andrade Júnior e Nogueira, (2017) e Araújo et al. (2019) as 
linhagens de feijão-caupi estão mais adaptadas a regiões mais quente e com índice pluviométrico próximo a $400 \mathrm{~mm} / \mathrm{ano}$.

O comprimento da vagem é de fundamental importância, pois vagens bem desenvolvidas influenciam diretamente no enchimento, massa e crescimento dos grãos de feijão-caupi e consequente produtividade.

Tabela 3 - Comparação das médias relacionadas às características agronômicas entre linhagens de feijão-caupi, cultivada nas condições do Recôncavo da Bahia, Brasil.

\begin{tabular}{|c|c|c|c|c|c|}
\hline Linhagens & $\begin{array}{c}\text { COMPV } \\
(\mathrm{cm})\end{array}$ & $\begin{array}{l}\text { M5V } \\
\text { (g) }\end{array}$ & $\begin{array}{l}\text { MG5V } \\
\text { (g) }\end{array}$ & $\begin{array}{c}\text { NG5V } \\
\left(n^{0}\right)\end{array}$ & $\begin{array}{c}\text { NGV } \\
\left(n^{0}\right)\end{array}$ \\
\hline MNC02-675F-4-9 & $17,16 \mathrm{~b}$ & $14,83 a$ & $11,45 \mathrm{a}$ & $57,25 \mathrm{a}$ & $11,45 a$ \\
\hline MNC02-675F-4-2 & $18,43 a$ & $16,63 a$ & $12,81 \mathrm{a}$ & $64,05 \mathrm{a}$ & $12,81 \mathrm{a}$ \\
\hline MNC02-675F-9-2 & $17,15 b$ & $14,93 a$ & $12,10 \mathrm{a}$ & $60,50 \mathrm{a}$ & $12,10 \mathrm{a}$ \\
\hline MNC02-675F-9-3 & $16,30 \mathrm{~b}$ & $10,76 \mathrm{~b}$ & $8,81 \mathrm{~b}$ & $42,55 b$ & $8,51 \mathrm{~b}$ \\
\hline $\mathrm{MNC} 02-676 \mathrm{~F}-3$ & $17,37 b$ & $15,53 \mathrm{a}$ & $11,11 \mathrm{a}$ & $55,55 \mathrm{a}$ & $11,11 \mathrm{a}$ \\
\hline MNC02-682F-2-6 & $17,40 \mathrm{~b}$ & $12,64 \mathrm{~b}$ & $9,46 b$ & $47,30 \mathrm{~b}$ & $9,46 b$ \\
\hline MNC02-683F-1 & $17,26 b$ & $12,96 \mathrm{~b}$ & $10,40 \mathrm{~b}$ & $52,00 \mathrm{~b}$ & $10,40 \mathrm{~b}$ \\
\hline MNC02-684F-5-6 & $17,39 b$ & $16,12 \mathrm{a}$ & $13,21 \mathrm{a}$ & $66,50 \mathrm{a}$ & $13,21 \mathrm{a}$ \\
\hline MNC03-725F-3 & $16,72 b$ & $12,36 \mathrm{~b}$ & $9,58 \mathrm{~b}$ & $47,90 \mathrm{~b}$ & $9,58 \mathrm{~b}$ \\
\hline MNC03-736F-7 & $20,00 \mathrm{a}$ & $16,32 \mathrm{a}$ & $12,92 \mathrm{a}$ & $64,60 \mathrm{a}$ & $12,92 \mathrm{a}$ \\
\hline MNC03-737F-5-1 & $15,88 \mathrm{~b}$ & $11,73 b$ & $9,62 b$ & $48,10 \mathrm{~b}$ & $9,62 b$ \\
\hline MNC03-737F-5-4 & $17,73 b$ & $12,43 b$ & $9,90 \mathrm{~b}$ & $49,50 \mathrm{~b}$ & $9,90 \mathrm{~b}$ \\
\hline MNC03-737F-5-9 & $15,27 b$ & $11,32 b$ & $9,47 \mathrm{~b}$ & $47,35 b$ & $9,47 \mathrm{~b}$ \\
\hline MNC03-737F-5-10 & $15,88 \mathrm{~b}$ & $11,73 b$ & $9,62 b$ & $47,33 b$ & $9,63 b$ \\
\hline MNC03-737F-5-11 & $17,61 b$ & $14,83 \mathrm{a}$ & $11,44 a$ & $52,46 \mathrm{~b}$ & $10,50 \mathrm{~b}$ \\
\hline MNC03-737F-11 & $16,82 b$ & $11,63 b$ & $9,57 \mathrm{~b}$ & $47,85 b$ & $9,57 \mathrm{~b}$ \\
\hline Cultivares & $\begin{array}{c}\text { COMPV } \\
\text { (cm) }\end{array}$ & $\begin{array}{c}\text { M5V } \\
\text { (g) }\end{array}$ & $\begin{array}{c}\text { MG5V } \\
\text { (g) }\end{array}$ & $\begin{array}{c}\text { NG5V } \\
\left(n^{0}\right)\end{array}$ & $\begin{array}{l}\text { NGV } \\
\left(\mathrm{n}^{0}\right)\end{array}$ \\
\hline BRS-TUMUCUMAQUE & $17,01 \mathrm{~b}$ & $12,12 b$ & $9,42 b$ & $47,10 \mathrm{~b}$ & $9,42 b$ \\
\hline BRS-CAUAMÉ & $16,36 \mathrm{~b}$ & $12,20 \mathrm{~b}$ & $9,27 \mathrm{~b}$ & $46,35 b$ & $9,27 \mathrm{~b}$ \\
\hline BRS-ITAIM & $15,83 b$ & $11,10 \mathrm{~b}$ & $9,82 \mathrm{~b}$ & $49,05 b$ & $9,81 \mathrm{~b}$ \\
\hline BRS-GUARIBA & $17,61 b$ & $13,13 b$ & $10,49 \mathrm{~b}$ & $52,45 b$ & $10,49 b$ \\
\hline
\end{tabular}

Médias seguidas por letras iguais na coluna não diferem entre si pelo teste Scott Knott com $5 \%$ de probabilidade. Comprimento médio das vagens (COMPV); massa de cinco vagens (M5V); massa dos grãos de cinco vagens (MG5V); número de grãos em cinco vagens (NG5V) e número de grãos por vagem (NGV).

Para as características da massa de cinco vagens (M5V) e massa dos grãos em cinco vagens (MG5V), foi observada a formação de dois grupos, com destaque para sete linhagens (MNC02-675F4-2, MNC03-736F-7, MNC02-684F-5-6, MNC02676F-3, MNC02-675F-9-2, MNCO3-737F-5-11 e MNC02-675F-4-9) formando o primeiro grupo. Também é importante destacar o desempenho inferior das cultivares avaliadas neste estudo (BRSTumucumaque, BRS-Cauamé, BRS-Itaim e BRSGuariba) que apresentaram resultados inferiores as linhagens. Essa informação é particularmente importante, pois demonstra potencial genético das linhagens para cultivo no Recôncavo da Bahia, além de haver a possibilidade de recombinação visando novo ciclo de seleção (Tabela 3). Estudos realizados com linhagens de feijão-caupi em Mato Grosso do Sul, Brasil apresentaram valores semelhantes para a massa de cinco vagens (Torres et al., 2015). Conforme Santos et al. (2014), os valores de (M5V) tem ligação direta com a maioria das variáveis agronômicas estudadas em Phaseolus vulgaris. Essa característica é importante em genótipos destinados à produção de grãos tanto verdes como secos, tendo em vista que ela mede a eficiência das linhagens na alocação de fotoassimilados para os grãos, servindo ainda como parâmetro para trabalhos de seleção em feijãocaupi (Freire Filho et al., 2005; Santos et al., 2014).

Fazendo o paralelo entre a massa dos grãos de cinco vagens deste trabalho com a massa de cem grãos dos trabalhos de Teixeira et al. (2010) no estado de Goiás, Silva e Neves (2011) no estado do Piauí, Santos e Lima (2015) no estado da Paraíba e Araújo et al. (2019) no estado do Ceará, todos estados situados no Brasil, fica evidente que não houveram grandes diferenças destes trabalhos com o trabalho avaliado no Recôncavo da Bahia, no entanto, apresentou superioridade aos trabalhos de Matoso et al. (2013) tanto na região do município de Dourados-MS, Brasil quanto em Botucatu-SP, Brasil. Sabe-se que a produtividade de grãos na cultura do feijão-caupi está diretamente correlacionada com as variáveis de produção 
(número de vagens por planta, número de grãos por vagem, rendimento de grãos por área, etc.) e dessa forma é possível indicar as linhagens mais promissoras com base nas características de produção, além disso, a depender das condições em que cada variável se apresente, essa produção pode aumentar ou diminuir e facilitar diretamente na estabilidade da produtividade dos grãos de feijãocaupi (Coimbra et al., 1999; Junior et al., 2005).

Analisando as variáveis de produção número de grãos em cinco vagens (NG5V) e número de grãos por vagens (NGV), é possível inferir que as linhagens MNC02-684F-5-6, MNC03-736F-7, MNC02-675F-4-2, MNC02-675F-9-2, MNC02675F-4-9 e MNC02-676F-3 se descartaram em relação às demais linhagens e cultivares utilizadas como controle (Tabela 3). A linhagem MNC02675F-9-3 apresentou valores inferiores às demais linhagens avaliadas, tanto para o número de grãos em cinco vagens quanto para o número de grãos por vagem (Tabela 3 ), apontando baixo potencial para recomendação aos produtores do Recôncavo da Bahia. Em média, as linhagens obtiveram resultados superiores ao trabalho de Torres et al. (2015), semelhante aos resultados de Teixeira et al. (2010), porém inferiores aos trabalhos de Silva e
Neves (2011) e Santos e Lima (2015) e Araújo et al. (2019), todos na região Nordeste do Brasil, comprovando assim que elevada umidade e pluviosidade superiores ao recomendado para essa cultura podem influenciar na produção de grãos do feijão-caupi.

Para um melhor entendimento sobre o comportamento em conjunto das linhagens e cultivares, foram realizadas análises de diversidade genética. Sendo possível a partir do mapa de calor visualizar a formação de quatro grupos, com destaque para quatro linhagens que formaram o primeiro grupo (Figura 1). O mapa de calor permite verificar que os resultados sejam mais robustos e eficientes para discriminar linhagens de qualquer cultura; além de ser mais fácil sua compreensão visual como forma de interpretação dos resultados. Rebouças et al. (2018) e Gonçalves et al. (2019) utilizaram a mesma ferramenta para selecionar genótipos resistentes a Fusarium oxysporum raça 1 em bananeiras. De acordo com os autores, o mapa de calor tem a vantagem de permitir que os híbridos de interesse sejam visualmente selecionados com base em uma escala de cores e progressão dos valores dos sintomas.

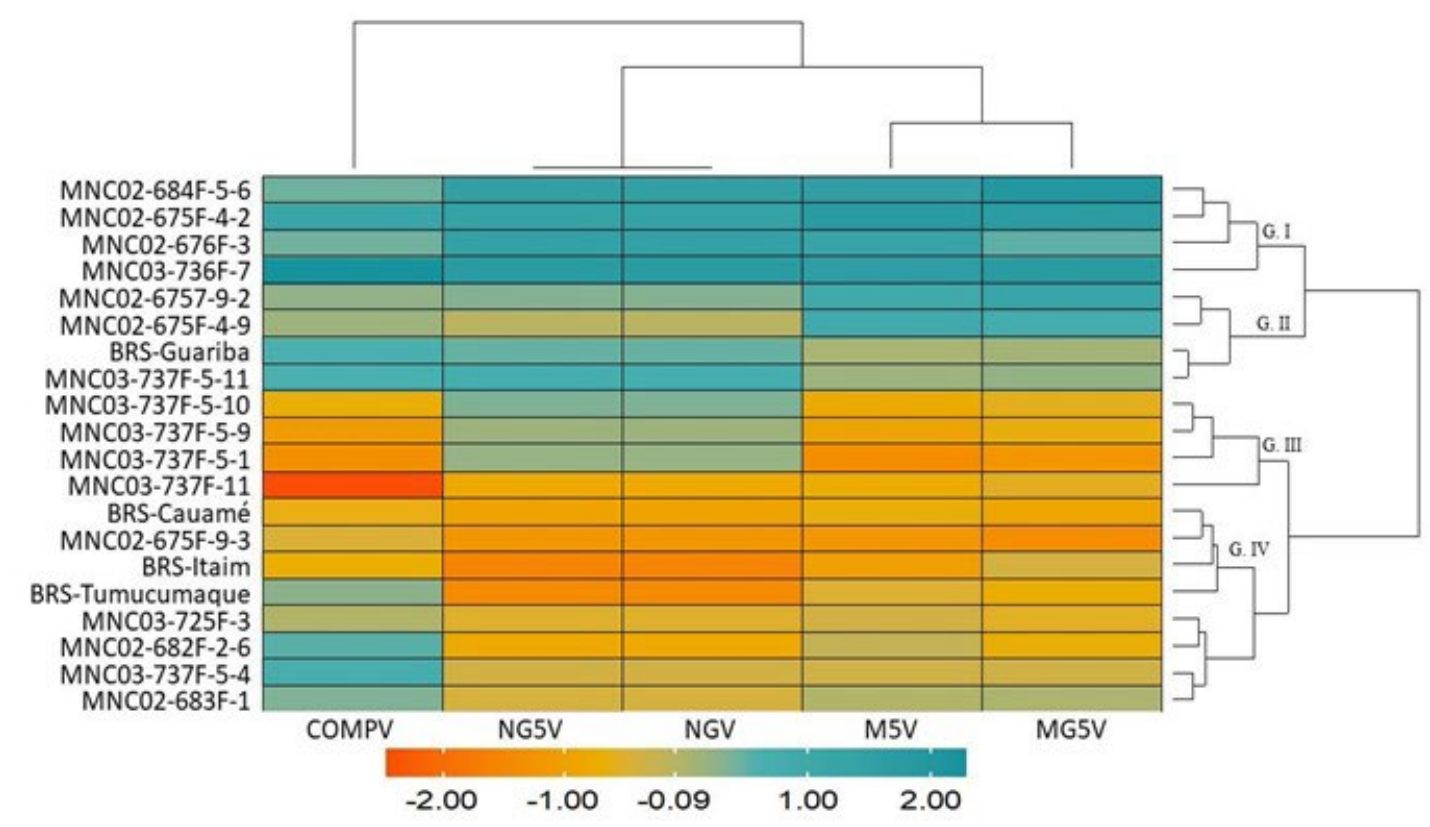

Figura 1 - Análise hierárquica de agrupamentos através do Mapa de calor (Heatmap) para as 16 linhagens e quatro cultivares de feijão-caupi produzidas nas condições do Recôncavo da Bahia, Brasil.

Conforme o dendograma (Figura 1) e gráfico de dispersão (Figura 2C) houve a formação de quatro grupos, o primeiro grupo (G.I) foi formado pelas linhagens MNCO2-684F-5-6, MNCO2-675F-4-2, $\mathrm{MNCO} 2-676 \mathrm{~F}-3$ e $\mathrm{MNCO} 3-736 \mathrm{~F}-7$ que se destacaram das demais linhagens avaliadas. $\mathrm{O}$ Grupo II formado por três linhagens (MNCO2-
675F-9-2, MNCO2-675F-4-9 e MNCO3-737F-511) e uma cultivar (BRS Guariba) apresentou resultados intermediários, enquanto a BRS Guariba se destacou entre todas as cultivares, apresentando desempenho satisfatório para o COMPV, NG5V e NGV (Figura 1). Os demais grupos (G. III e G. IV), correspondentes a $60 \%$ dos genótipos exibiram 
valores inferiores em relação aos genótipos do G. I e G. II.

Bertini et al. (2010) avaliando o desempenho de linhagens superiores de feijão-caupi em FortalezaCE observaram que os genótipos avaliados se comportaram de diferentes formas de acordo principalmente pela massa dos grãos, coloração das sementes e número de grãos das vagens, corroborando com os resultados aqui apresentados. Santos et al. (2014) trabalhando com 20 genótipos de feijão-caupi no Mato Grosso, Brasil, conseguiram através de diferentes métodos de agrupamentos influir que houve divergência genética entre os genótipos, formando cinco grupos divergentes, corroborando com os achados deste trabalho no recôncavo da Bahia.

Os dois primeiros componentes principais ( $\mathrm{PC} 1$ e PC2), referentes aos cinco caracteres agronômicos avaliados, viabilizaram a construção de uma dispersão gráfica, pelo fato dos dois componentes explicarem $93,0 \%$ da variância total dos dados (Figura 2C). As cargas fatoriais com maior potencial para descriminação com PC1 foram M5V e MG5V e para o PC2 COMPV (Figura 2A).

Através da dispersão gráfica em função dos dois componentes principais é evidente a formação dos grupos das linhagens de feijão-caupi bem distintos, com destaque principalmente para as linhagens MNCO3-736F-7 e MNCO2-675F-4-2 que foram influenciadas principalmente pelo COMPV e M5V (Figuras 2B e C). Já as linhagens MNCO3-737F-510, MNCO3-737F-5-9, MNCO3-737F-11 e MNCO3-737F-5-1 exibiram o menor desempenho entre as linhagens testadas (Figura 2C).
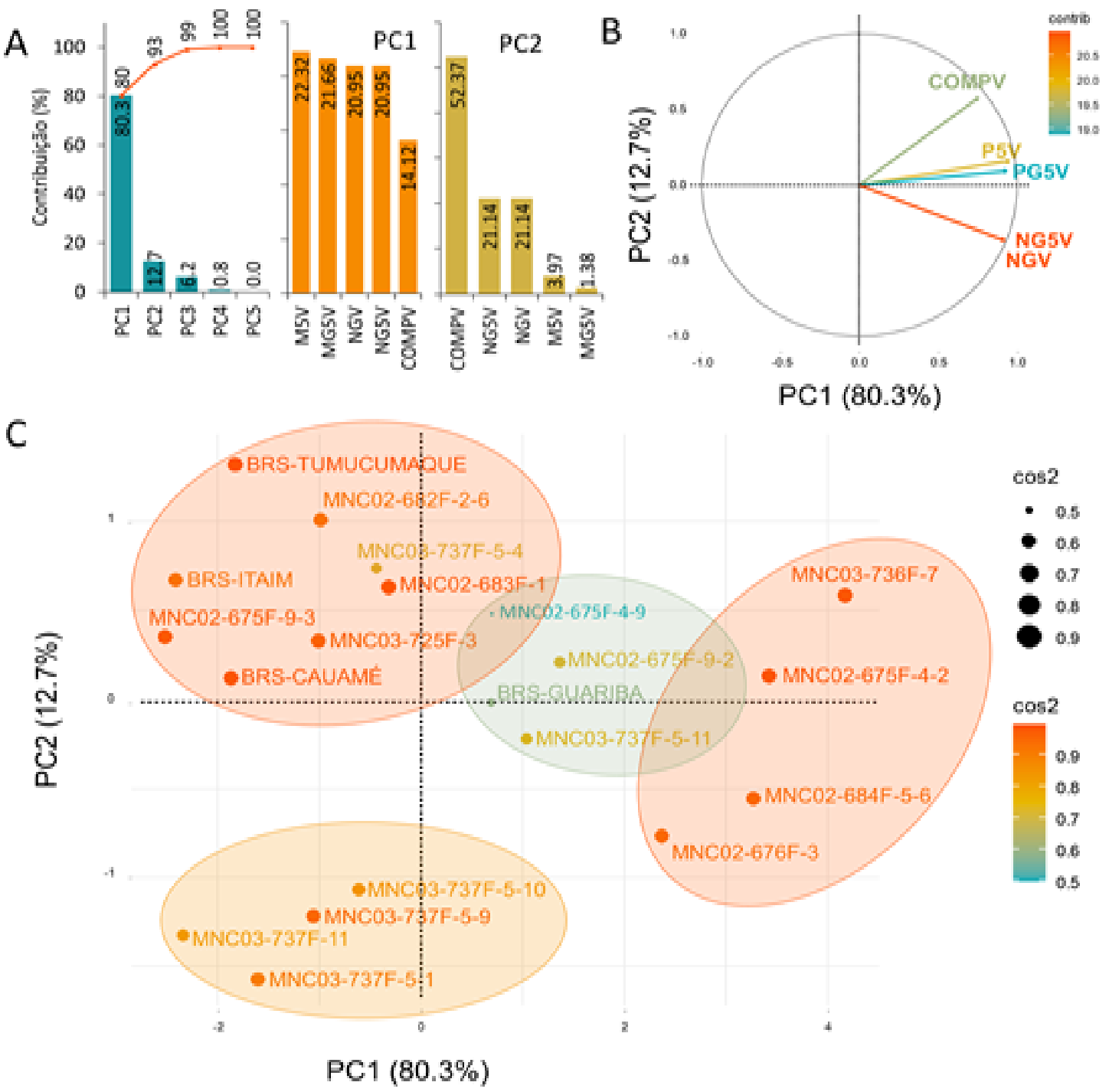

Figura 2 - Gráfico de dispersão em função dos dois primeiros componentes PC1 e PC2 das 16 linhagens e quatro cultivares comerciais de feijão-caupi nas condições do Recôncavo da Bahia, Brasil. 
Estudo conduzido por Santos et al. (2016) avaliando a produtividade de 20 cultivares de feijão-caupi, observaram que as cultivares BRSTumucumaque e BRS Guariba compuseram o mesmo grupo, divergindo dos resultados apresentados neste trabalho. Souza et al. (2007) e Santos et al. (2014), observaram diferentes formações de grupos em seus trabalhos, essa variação pode estar ligada aos diferentes progenitores de cada linhagem de feijão estudada e a interação genótipo ambiente.

Conforme Ramalho et al. (2012), o poder de interação dos genótipos com o ambiente é caracterizado quanto ao comportamento das raças, linhagens ou cultivares não são consistentes, com isso, a resposta de cada genótipo é específica e diferente de outros genótipos de acordo com as mudanças ocorridas em cada ambiente. Diante disso, estudos dessa magnitude são cruciais para o melhoramento de plantas, pois fornecem informações sobre o comportamento de cada genótipo em diferentes variações do ambiente.

Para o lançamento de uma cultivar é necessário à execução de várias etapas, dentre elas a avaliação da estabilidade fenotípica junto ao grupo genético, favorece na inferência dos efeitos da interação genótipo versus ambiente, sendo uma prática muito utilizada pelos melhoristas antes da sua recomendação.

Todas as correlações entre as características produtivas de linhagens e cultivares de feijão-caupi foram positivas. Sendo que sete das dez correlações possíveis foram altamente significativas $(\mathrm{p} \leq 0.01)$ e correlação perfeita $(R=1)$ foi observara entre NG5V e NGV, estas variáveis estão diretamente ligadas e são os componentes que mais influenciam na produção e seleção de cultivares mais produtivas (Figura 3).

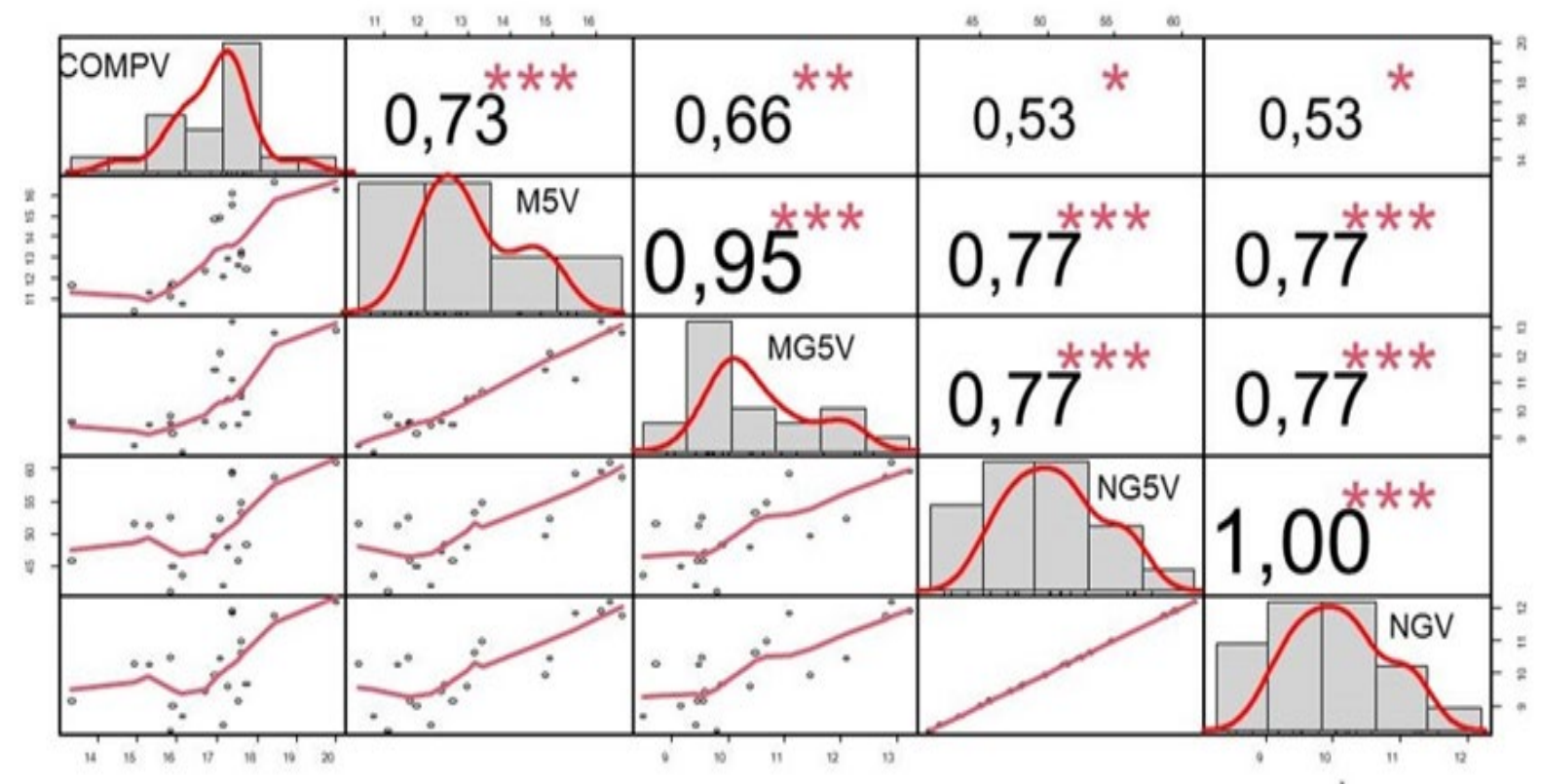

Figura 3 - Correlação fenotípica de Pearson na diagonal superior para as cinco variáveis biométricas e na diagonal inferior gráfico de dispersão com a relação entre os caracteres na diagonal central, apresentando histograma com intervalos de classe e linha de distribuição normal dos dados, $*$, ** e *** indicando significância a $5 \%, 1 \%$ e $>1 \%$ pelo valor de $P$.

As menores correlações foram registradas entre o comprimento das vagens (COMPV) com NG5V e NGV (Figura 3). Conforme Correa et al. (2012), as mais baixas correlações genotípicas e fenotípicas podem ser explicadas pelo processo gradativo de desidratação dos grãos no campo após sua maturação fisiológica e no processo de colheita e armazenamento. Tanto Matos Filho et al. (2009) quanto Bertini et al. (2010) obtiveram os maiores valores de correlações positivas entre as variáveis de produção, porém quando se analisa seus dados quanto ao número de sementes, é perceptível a sua inferioridade quando comparado com as correlações reportadas no presente estudo. Correa et al. (2012) trabalhando com feijão dos tipos prostrado e semi-prostrado na região Centro-Oeste do Brasil, obtiveram resultados de correlação distintos do presente trabalho, onde os mesmos apresentaram resultados de comprimento das vagens com massa de cinco vagens abaixo de $R=$ 0,40 , considerado baixo para esse tipo de estudo. Já Silva e Neves (2011) encontraram valores de 
correlação bem inferiores quando comparados ao deste trabalho, podendo ser explicado pela baixa pluviosidade e altas temperaturas onde foi avaliado seu experimento, influenciando diretamente em algumas variáveis em estudo.

A partir dos avanços reportados neste estudo é possível inferir elevada variabilidade genética entre as linhagens de feijão-caupi avaliadas e elencar pelo menos duas linhagens (MNC02-675F-4-2 e MNC03-736F-7) com potencial agronômico para produção nas condições do Recôncavo da Bahia, pois se mostraram superiores para todas as características avaliadas em relação às cultivares (controle), contudo, avaliações em outras condições e em diferentes ciclos devem ser priorizadas para confirmação dessas informações e posterior recomendação para os produtores de feijão-caupi do Recôncavo da Bahia.

\section{CONCLUSÕES}

As linhagens MNC02-675F-4-2 e MNC03$736 \mathrm{~F}-7$ são recomendadas para produção do feijãocaupi nas condições do Recôncavo da Bahia, devido ao seu elevado potencial agronômico e desempenho superior em relação às cultivares utilizadas no controle.

De acordo as análises apresentadas, houve a formação de quatro grupos distintos, podendo inferir que as linhagens selecionadas possuem os mesmos ancestrais em comum.

\section{REFERÊNCIAS BIBLIOGRÁFICAS}

Agovino M, Casaccia M, Ciommi M, Ferrara M, Marchesano K. Agriculture, climate change and sustainability: The case of EU-28. Ecological Indicators, v.105, p.525-543, 2019. https://doi.org/10.1016/j.ecolind.2018.04.064

Akibode S, Maredia M (2012) Global and regional trends in production, trade and consumption of food legume crops. Report submitted to CGIAR Special panel on impact assessment, Department of Agricultural, Food and Resource Economics Michigan State University.

https://doi.org/10.22004/ag.econ.136293

Araújo LBR, Pinheiro MS, Fiege LBC, Bertini CHCM, Dovale JC. Agronomic potential and genetic diversity of landraces of cowpea of the state of Ceará. Revista Caatinga, v.32, n.3, p.698-708, 2019. https://doi.org/10.1590/1983$21252019 \mathrm{v} 32 \mathrm{n} 314 \mathrm{rc}$

Barros, MA, Rocha MM, Gomes RLF, Silva KJD, Neves AC. Adaptabilidade e estabilidade produtiva de feijao-caupi de porte semiprostrado. Pesquisa Agropecuária Brasileira, Brasília, v.48, n.4, p.403-410, 2013. https://doi.org/10.1590/S0100-204X2013000400008

Bastos EA, Andrade Júnior AS, Nogueira CCP. Cultivo de Feijão-Caupi. Disponível em: $<$ https://ainfo.cnptia.embrapa.br/digital/bitstream/item/161199/1/SistemaProducaoCaupiCapituloIrrigacao.pdf $>$. Acesso em: 05 Dez. 2020.
Bertini CHCM, Almeida WS, Silva APM, Silva JWL, Teofilo EM. Análise multivariada e índice de seleção na identificação de genótipos superiores de feijão-caupi. Acta Scientiarum Agronomy, Maringá, v.32, n.4, p.613-619, 2010. https://doi.org/10.4025/actasciagron.v32i4.4631

Bocchiola D, Brunetti L, Soncini A, Polinelli F, Gianinetto M (2019) Impact of climate change on agricultural productivity and food security in the Himalayas: a case study in Nepal. Agricultural Systems, v.171, p.113-125, 2019. https://doi.org/10.1016/j.agsy.2019.01.008

Coimbra JLM, Guidolin AF, Carvalho FIF, Coimbra SMM, Marchioro VS. Análise de trilha I: Análise do rendimento de grãos e seus componentes. Ciência Rural, Santa Maria, v.29, n.2, p.213-218, 1999.

COMPANHIA NACIONAL DE ABASTECIMENTO - CONAB. Acompanhamento da safra brasileira de grãos, v.6 Safra 2018/19 - Nono levantamento, Brasília, p.1-113, janeiro 2021.

FAO. Food and agriculture organization of the united nations statistics division, 2014. Disponível em: $<\mathrm{http}: / /$ faostat3.fao.org/browse/Q/QC/E, 2014>. Acesso em: 01 nov. 2020.

Correa AM, Ceccon G, Correa CMA, Delben DS. Estimativas de parâmetros genéticos e correlações entre caracteres fenológicos e morfoagronômicos em feijão-caupi. Revista Ceres, v.59 n.1, Viçosa, 2012.

https://doi.org/10.1590/S0034-737X2012000100013

Dantas APJ, Holanda GC, Rolim RR, Ferreira LT, Nascimento NFF, Araújo HFP. Evaluation of two cowpea (Vigna unguiculata (L.) walp.) genotypes under rainfed farming with low rainfall. Revista Brasileira Meio Ambiente, v.7, n.3, p.58-69, 2019. https://doi.org/10.5281/zenodo. 3575407

Devi CB, Kushwaha A, Kumar A. Sprouting characteristics and associated changes in nutritional composition of Cowpea (Vigna unguiculata). Journal of Food Science and Technology, v.52, p.6821-6827, 2015. https://doi.org/10.1007/s13197-015-1832-1

Freire Filho FR, Lima JAA, Ribeiro VQ. Feijão-caupi: Avanços tecnológicos. Brasília: Embrapa Informação Tecnológica; Teresina: Embrapa Meio-Norte, p.519, 2005.

Freire Filho FR, Ribeiro VQ, Rocha MM, Silva KJD, Nogueira MSR, Rodrigues EV. Caracterização de pólos de produção da cultura de feijão-caupi no estado o Piauí. Embrapa Meio Norte, 2007, p.28. (Documento, 100).

Gonçalves ZS, Haddad H, Amorim VBO, Ferreira CF, Oliveira SAS, Amorim EP. Agronomic characterization and identification of banana genotypes resistant to Fusarium wilt race 1. European Journal of Plant Pathology, v.155, p.1093-1103, 2019. https://doi.org/10.1007/s10658-01901837-5

Júnior EUR, Lemos LB, Silva TRB. da. Componentes da produção, produtividade de grãos e características tecnológicas de cultivares de feijão. Revista Bragantia, Campinas, v.64, n.1, p.75-82, 2005. https://doi.org/10.1590/S000687052005000100008

Locatelli, VER, Medeiros RD, Smiderle OJ, Albuquerque JAA, Araujo WF, Souza KTS. Components of production, productivity and efficiency of irrigation of bean-cowpea in 
the "Cerrado" of Roraima. Revista Brasileira de Engenharia Agrícola e Ambiental, v.18, n.6, p.574-580, 2014. http://dx.doi.org/10.1590/S1415-43662014000600002

Matos Filho CHA, Gomes RLF, Rocha MM, Freire Filho FR, Lopes ACA. Potencial produtivo de progênies de feijãocaupi com arquitetura ereta de planta. Ciência Rural, v.39 n.2, p.348-354, 2009.

http://dx.doi.org/10.1590/S0103-84782009000200006

Matoso AO, Soratto RP, Ceccon G, Figueiredo PG, Neto ALN. Desempenho agronômico de feijao-caupi e milho semeados em faixas na safrinha. Pesquisa agropecuária brasileira, Brasília, v.48, n.7, p.722-730, 2013. https://doi.org/10.1590/S0100-204X2013000700004

R Core Development Team. (2016). R: A language and environment for statistical computing, reference index version 2.12.1. ISBN 3-900051-07-0. Viena: R Foundation for Statistical Computing.

Ramalho MAP, Abreu AFB, Santos JB, Nunes JAR. Aplicações da genética quantitativa no melhoramento de plantas autógamas. Lavras: Ufla, p.522, 2012.

Rebouças TA, Haddad F, Ferreira CF, Oliveira SAS, Ledo CAS, Amorim EP. Identification of banana genotypes resistant to Fusarium wilt race 1 under field and greenhouse conditions. Scientia Horticulturae, v.239, p.308-313, 2018. https://doi.org/10.1016/j.scienta.2018.04.037

Santos JAS, Teodoro PE, Correa AM, Soares CMG, Ribeiro LP, Abreu HKA. Desempenho agronômico e divergência genética entre genótipos de feijão-caupi cultivados no ecótono Cerrado/Pantanal. Revista Bragantia, v.73, n.4, p.377382, 2014. https://doi.org/10.1590/1678-4499.0250

Santos D, Lima LKS. Avaliação agronômica de variedades de feijão-caupi em cultivo de sequeiro no município de Coremas-PB. Revista Verde, v.10, n.1, p.218-222, 2015. http://dx.doi.org/10.18378/rvads.v10i1.2950

Santos A, Ceccon G, Teodoro PE, Correa AM, Alvarez RCF, Silva JF, Alves VB. Adaptabilidade e estabilidade de genótipos de feijão caupi ereto via REML/BLUP e GGE Biplot. Revista Bragantia v.75 n.3 Campinas, 2016.

https://doi.org/10.1590/1678-4499.280

Silva JAL, Neves JA. Componentes de produção e suas correlações em genótipos de feijão-caupi em cultivo de sequeiro e irrigado. Revista Ciência Agronômica, v.42 n.3, p.702-713, Fortaleza, 2011. https://doi.org/10.1590/S180666902011000300017

Silva FHAD, Torres SB, Carvalho SMC, Bai M, Lopes WDAR. Physical and physiological attributes of saved cowpea seeds used in the brazilian semi-arid region. Revista Caatinga, v.32, n.1, p.113-120, 2019. https://doi.org/10.1590/1983-21252019v32n112rc

Sousa JLM. Rocha MM, Silva KJD, Neves AC, Sousa RR. Potencial de genótipos de feijão-caupi para o mercado de vagens e grãos verdes. Pesquisa Agropecuária Brasileira, v.50, n.5, p.392-398, 2015. http://dx.doi.org/10.1590/S0100-204X2015000500006

Souza LS, Souza LD. Caracterização físico-hídrica de solos da área do Centro Nacional de Pesquisa Mandioca e Fruticultura Tropical. Boletim de Pesquisa e Desenvolvimento, $\mathrm{n}^{\circ} 20$, Embrapa Mandioca e Fruticultura, p.56, 2001. ISSN 1516-5604
Souza CLC, Lopes ACA, Gomes RLF, Rocha MM, Silva EM. Variability and correlations in cowpea populations for green-grain production. Crop Breeding and Applied Biotechnology, ed.7, p.262-269, 2007.

Teixeira IR, Silva GC, Oliveira JPR, Silva AG, Pelá A. Desempenho agronômico e qualidade de sementes de cultivares de feijão-caupi na região do cerrado. Revista Ciência Agronômica, v.41, n.2, p.300-307, 2010. https://doi.org/10.1590/S1806-66902010000200019

Torres FE, Teodoro PE, Sagrilo E, Ceccon G, Correa AM. Interação genótipo $\mathrm{x}$ ambiente em genótipos de feijãocaupi semiprostrado via modelos mistos. Revista Bragantia, v.74, n.3, p.255-260, 2015 https://doi.org/10.1590/1678-4499.0099 